Marquette University

e-Publications@Marquette

Mechanical Engineering Faculty Research and

Publications

Mechanical Engineering, Department of

7-15-2018

\title{
Pore-resolving Simulation of Char Particle Gasification Using Micro-CT
}

Greg Hingwah Fong

Marquette University

Scott Jorgensen

Marquette University

Simcha L. Singer

Marquette University, simcha.singer@marquette.edu

Accepted version. Fuel, Vol. 224 (July 15, 2018): 752-763. DOI. (C) 2018 Elsevier. Used with permission. 
Marquette University

e-Publications@Marquette

\title{
Mechanical Engineering Faculty Research and Publications/College of Engineering
}

This paper is NOT THE PUBLISHED VERSION; but the author's final, peer-reviewed manuscript. The published version may be accessed by following the link in th citation below.

Fuel, Vol. 224 (July 15, 2018): 752-763. DOI. This article is (C) Elsevier and permission has been granted for this version to appear in e-Publications@Marquette. Elsevier does not grant permission for this article to be further copied/distributed or hosted elsewhere without the express permission from Elsevier.

\section{Pore-resolving Simulation of Char Particle Gasification Using Micro-CT}

\author{
Greg H. Fong
}

Department of Mechanical Engineering, Marquette University, Milwaukee, WI Scott Jorgensen

Department of Mechanical Engineering, Marquette University, Milwaukee, WI

Simcha L. Singer

Department of Mechanical Engineering, Marquette University, Milwaukee, WI

\footnotetext{
Abstract

Understanding the interaction between transport, reaction and morphology at the scale of individual char particles is important for optimizing solid fuel gasification and combustion processes. However, most particlescale models treat porous char particles as an effective porous continuum, even though the presence of large, irregular macropores, voids and fractures render such upscaled treatments mathematically invalid, and the models non-predictive. A new modeling framework is therefore proposed to elucidate the impact of morphology on char particle gasification and combustion. A pore-resolving, transient, three-dimensional simulation for gasification of a realistic coal char particle is developed based on X-ray micro-computed
} 
tomography (micro-CT). The large macropores and voids resolved by micro-CT are explicitly represented in the particle's geometry and conservation equations based on first principles are solved in those regions. Upscaled, effective-continuum equations are applied only within the micro- and meso-porous grains surrounding the voids, where such equations are mathematically appropriate. To assess the impact of the realistic particle morphology, a second model which employs effective-continuum equations everywhere and assumes spherical symmetry is also developed for a particle having the same initial mass, volume, porosity, surface area and equivalent diameter as the pore-resolving model. The results indicate that large, irregular voids enhance mass transport throughout the particle and affect its overall conversion behavior when reactions occur under intraparticle diffusion control.

\section{Keywords}

Pore-resolving, micro-CT, Coal, Char, Gasification, Combustion, Nomenclature

\section{Nomenclature}

\section{A pre-factor exponential}

B permeability

D diffusion coefficient

D diameter

$E_{a}$ activation energy

$\mathrm{H}$ enthalpy

$\overline{\text { J diffusion flux }}$

$\mathrm{K}$ thermal conductivity

MW molecular weight

$M$ mass

P pressure

$\mathrm{R}_{\mathrm{i}}$ rate of production of $i^{\text {th }}$ Species

$R_{u}$ universal gas constant

$R$ radius

$S$ surface area per unit volume

$\mathrm{S}_{\mathrm{g}}$ specific surface area (per unit mass)

$S_{f}^{h}$ fluid enthalpy source

$S_{m}$ mass source term

$T$ temperature

T time

$\vec{v}$ velocity

$\mathrm{V}$ atomic diffusion volume

$\checkmark$ volume

$\mathrm{X}$ conversion

$x$ mole fraction

$Y$ mass fraction

$\varepsilon$ porosity

$\vartheta$ stoichiometric coefficient

$\mu$ dynamic viscosity

$\rho$ density

$\tau$ tortuosity

$\overline{\bar{\tau}}$ stress tensor 
$\Psi$ pore structure parameter

$\eta$ effectiveness factor

$\mathcal{R}$ reaction rate (intrinsic)

Subscripts

2D effective continuum model

3D pore-resolved model

$c$ carbon

eff effective

equiv equivalent (diameter)

$\mathrm{i} \mathrm{i}^{\text {th }}$ Species

j jth Species or Reaction

knud Knudsen

m mixture

MPS microporous solid

$\mathrm{n}$ reaction order

o initial

p pore

rxn reaction

s solid

scale scale factor

$\mathrm{t}$ true (density)

$T$ thermal

Tot total

\section{Introduction}

The gasification and combustion behavior of individual coal char particles affects important outputs at the reactor-scale, such as carbon conversion [1] and temperature [2]. In entrained flow gasification [3] and combustion [4] processes, char particles often react under zone II conditions, in which both reaction and transport through the particle's pore structure influence the char consumption rate. For zone II conditions, it has been convincingly demonstrated that particle morphology has a strong impact on conversion rates and cannot be accurately simulated assuming spherical, homogeneous particles [5]. Therefore, strategies to optimize thermochemical conversion processes via improved reactor design and operating conditions require a fundamental understanding of the interplay between reaction, transport and morphology at the scale of the individual porous char particles.

Since it is impossible to solve conservation equations for reaction and transport within the actual char structure and its myriad pores, physics and chemistry at the scale of these geometrical heterogeneities are typically averaged, or "upscaled". Upscaling (e.g. via homogenization [6] or volume averaging [7]) transforms equations based on first principles and pore-scale geometries into computationally tractable, effective-continuum equations, which contain effective properties that are dependent on the porous medium's characteristics.

For mathematical validity and accuracy, upscaling methods require a separation of length scales [8], [9]. This implies that the characteristic length-scale of the geometrical heterogeneities (e.g. pores) must be significantly smaller than that of the system (e.g. particle), as well as the characteristic length of the physical processes being studied (e.g. concentration gradients) [9]: 
(1a) $L_{\text {pores }}<<L_{\text {particle }}$

(1b) $L_{\text {pores }}<<L_{\text {gradient }}$

Inequality (1a) implies that upscaling is meaningful only if there exists a statistically significant sample of heterogeneities over which to average (a representative volume element), but treating heterogeneities with sizes approaching that of the macroscopic domain as an effective-continuum is not valid. Similarly, if the characteristic length-scale of the concentration or temperature gradients becomes nearly as small as the lengthscale of the geometric heterogeneities, local averaging is impossible and inequality $(1 \mathrm{~b})$ is violated.

For char particle gasification and combustion, the length-scale constraints are particularly acute [8], [10]. Across a range of coals and devolatilization conditions, char particles contain large macro-pores, voids and fractures with dimensions that approach the scale of the particles themselves [10], [11]. This results in violation of the length-scale constraints and renders an effective-continuum treatment invalid and non-predictive. Application of an effective-continuum model when the length-scale constraints are not satisfied can result in the failure to predict the extent of heterogeneous reactions [12], mixing [13] and localized phenomena such as hotspots [14].

Despite their general inapplicability, the vast majority of spatially-resolved char particle gasification and combustion models simply ignore the length-scale constraints and employ spherically symmetric, effectivecontinuum models. Although such an approach might be applicable to synthetic char particles, which are spherical, uniformly porous and lack large voids [15], or to hypothetical spherical particles with a single spherical void [16], the effective-continuum approach is not applicable to realistic void and large macropore morphologies. For this reason, effective-continuum simulations often require adjustable parameters to match experimental data.

This issue has been recognized by several investigators and has led to discrete representations of porous char particles, using Monte Carlo or random walk methods to analyze char conversion [8], [10], [17], [18]. However, the fidelity with which discrete networks represent actual char particles is questionable, and the discrete approach is difficult to combine with models of other physical and chemical processes, such as mass

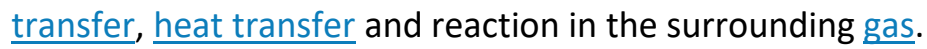

Two recent studies have developed simulations of char particle combustion that resolve large pores/voids, but both are based on idealized pore structures and treat the char surrounding the voids as completely non-porous. Richter et al. simulated oxy-combustion of a porous coal char particle by treating the particle as an agglomerate of 185 monodisperse, non-porous spheres, each with a diameter of $25 \mu \mathrm{m}$ [19]. The void-space between the spheres was meshed and the reacting flow problem was solved using ANSYS FLUENT. Calculations for the entire agglomerate were compared to a non-porous particle, and enhanced reaction rates were observed for the agglomerate. The agglomerate model was later used to calculate drag coefficients and Nusselt numbers for porous particles [20]. Xue et al. used a very similar approach to study oxy-combustion of particles with coneshaped pores extending from the surface toward the particle center [21]. The char surrounding the idealized pores was also treated as non-porous.

In the context of non-reacting simulations, micro-CT has been used to image coal macro-pores larger than $13.85 \mu \mathrm{m}$ for flow simulations using COMSOL [22]. Ciesielski and coworkers used confocal scanning laser microscopy on sections of wood particles to obtain microstructural parameters characteristic of the pore structure [23]. The data was used in a constructive solid geometry algorithm to generate three particle geometries which were used in simulations of heat transfer and mass transfer using COMSOL [23], [24]. It was concluded that intra-particle transport is not adequately represented using effective-continuum models. 
This paper presents the first pore-resolving, reacting flow simulation for char particle conversion based on a realistic char particle. The approach resolves large pores and voids, circumventing difficulties associated with upscaling large heterogeneities, which have prevented previous effective-continuum treatments from being truly predictive. A second novelty of the current approach is the combination of the void-resolving simulation with an effective-porous-continuum treatment of the micro/meso-porous grains surrounding the resolved pores, in contrast to [19], [21], where the char grains surrounding the idealized resolved pores were assumed to be completely non-porous. In the transient, 3-D simulation approach outlined below, the large macropores and voids are explicitly represented in the particle's geometry using micro-CT, and conservation equations based on first principles are solved in those regions, as shown in Fig. 1. Effective-continuum equations are employed only within the micro/meso-porous grains (referred to hereafter as "microporous", although these regions contain micro-, meso- and macro-pores), where they are mathematically appropriate and where the length-scale constraints (Eq. (1a), (1b)) for upscaling are satisfied (see Fig. 1). While equally applicable to combustion, the modeling framework is demonstrated here for entrained flow gasification conditions.

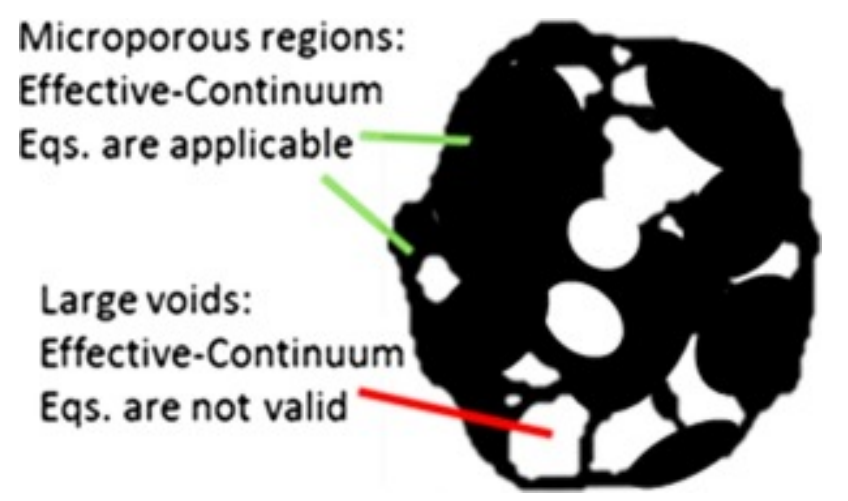

Fig. 1. Schematic of char particle showing resolved pores and voids, as well as micro/meso-porous grains.

The methods of char production and micro-CT imaging are described in Section 2. Development of the poreresolving simulation and the effective-continuum simulation are presented in Section $\underline{3}$, and a procedure ensuring that the respective particles have identical initial mass, volume, porosity, surface area and equivalent diameter is discussed. A comparison of results from the pore-resolving and effective-continuum simulations is presented in Section $\underline{4}$, and conclusions are discussed in Section $\underline{5}$.

\section{Materials and methods}

\subsection{Char production}

A bituminous coal was employed in this study. The particles were pyrolyzed in an electrically heated furnace at a heating rate of $100{ }^{\circ} \mathrm{C} / \mathrm{min}$ up to $800^{\circ} \mathrm{C}$ in an oxygen-free environment. The temperature was held at $800^{\circ} \mathrm{C}$ for $20 \mathrm{~min}$, after which the char was cooled to room temperature prior to collection. In this initial study, $850 \mu \mathrm{m}$ diameter coal particles were employed due to the resolution of the micro-CT, as discussed in Section 2.2.

Although the pyrolysis conditions and particle size are not typical of entrained flow gasifiers, a scaling procedure described in Section $\underline{3.1}$ is used to modify the particle size prior to gasification simulations.

\subsection{Micro-CT imaging}

Several char particles were attached to a cylindrical holder with double-sided adhesive and loaded into the micro-CT. A Hamamatsu L9181-02 micro-focal X-ray source and a Varian 2520DX flat panel X-ray detector was used for 3-D micro-CT imaging. The micro-CT is capable of a maximum resolution of $15 \mu \mathrm{m}$. A voxel size of $20 \mu \mathrm{m}$ 
was used in this study, which was sufficient to resolve the large features (voids, pores, irregularities) within the $850 \mu \mathrm{m}$ particles.

Following micro-CT imaging, reconstruction and image processing were used to generate stacks of twodimensional TIFF files for individual char particles. A stack of images representing an individual particle was exported to Simpleware ScanIP (Synopsys, Mountain View, USA) for segmentation and image processing [25], where the particle was visualized in three dimensions and the domain was cropped. To partition the geometry into resolved pores and microporous solid, segmentation was performed using ScanIP's connectivity tool and a binary greyscale criterion. The geometry was then smoothed using recursive Gaussian filtering to reduce sharp edges and the likelihood of complex meshing elements being generated. The char particle shown in Fig. 2(a) was used in the pore-resolving simulation described in Section $\underline{3}$.
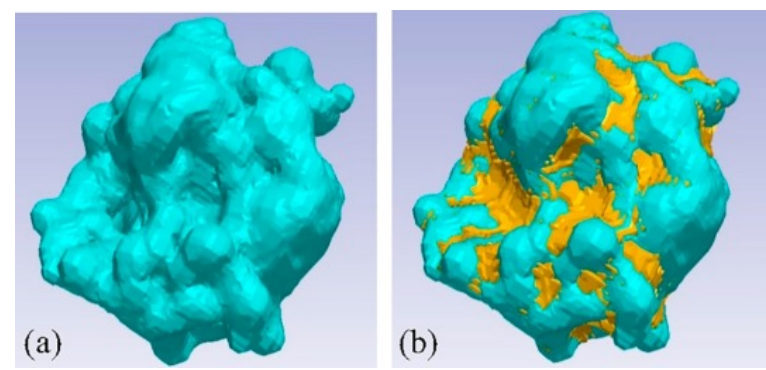

Fig. 2. (a) Reconstructed char particle, and (b) the particle with resolved voids (gold) delineated.

\section{CFD simulations}

\subsection{Char particle characterization}

To analyze the impact of char particle morphology, two models were developed: a three-dimensional, poreresolving simulation based on micro-CT imaging and a spherically-symmetric, effective-continuum simulation. To ensure a valid comparison between the pore-resolving and effective-continuum particles, the initial mass, total volume, total porosity, total internal surface area, and equivalent diameter had to be identical for both particles.

The volumes of the resolved voids and the surrounding microporous regions were first quantified for the poreresolving model. The volume attributed to the resolved pores and voids accounts for dead-end and through pores, including major concavities [26]. Consistent with this definition, the volume of the resolved voids is calculated using ScanIP's "Close" tool, forming the distinct gold regions shown in Fig. 2(b). The volumes of both the resolved voids, $V_{\text {resolvedvoids, }}$, and the surrounding microporous solid regions, $V_{\text {micro-solid }}$, were then measured. The total volume of the resolved particle (subscript 3D) is the sum of the two volumes:

\section{(2) $V_{3 D, \text { tot }}=V_{\text {resolvedvoids }}+V_{\text {micro-solid }}$}

Relatively large 850 um char particles were used in this study due to the resolution of the micro-CT, while char particles in entrained flow gasifiers typically have diameters of $100 \mu \mathrm{m}$. To conform to the entrained flow gasification conditions to be simulated, the particle geometry was isotropically scaled to a diameter of $100 \mu \mathrm{m}$. Because the goal of this work is to demonstrate a new simulation framework and to assess the impact of irregular morphological features, the current approach assumes the morphology of $100 \mu \mathrm{m}$ and $850 \mu \mathrm{m}$ particles are similar.

To perform the scaling, an equivalent diameter [27] was first calculated for the pore-resolving particle:

(3) $d_{3 D, \text { equiv }}=\left(\frac{6 V_{3 D, t o t}}{\pi}\right)^{\frac{1}{3}}$ 
Then a scaling factor, $R_{\text {scale }}$, was calculated, to ensure that the effective-continuum (subscript 2D) and poreresolving models had identical equivalent diameters,

$$
\text { (4) } R_{\text {scale }}=\frac{d_{2 D}}{d_{3 D, \text { equiv }}}
$$

where the spherical, effective-continuum particle's actual diameter, $d_{2 D \text {, }}$ is the same as its equivalent diameter, and equals $100 \mu \mathrm{m}$. The pore-resolving particle was scaled isotropically to ensure that the equivalent diameters were identical. Examination of Eq. (3) demonstrates that if the equivalent diameters are identical, the total volume of the respective particles are also identical. This was confirmed by measurements using the software.

The pore-resolving model contains a portion of its porosity within the resolved voids (the gold regions of Fig. 2b) and a portion of its porosity within the microporous grains (the blue regions of Fig. 2 b) surrounding those voids. To ensure that the total initial porosity is identical in both models, the porosity of the unresolved, microporous regions will necessarily be smaller than the uniformly distributed porosity of the effective-continuum model. To determine the initial porosity of the microporous grains in the pore-resolving model, $\varepsilon_{\text {micro-solid, } 0 \text {, the total }}$ porosity is written as a function of the porosity of the resolved voids, $\varepsilon_{\text {voids }}$, and the porosity of the microporous solid regions:

(5) $\varepsilon_{3 D, T o t, 0}=\varepsilon_{\text {voids }}+\left(1-\varepsilon_{\text {voids }}\right) \varepsilon_{\text {micro-solid }, 0}$

The porosity of the resolved voids is calculated from the measured volumes,

(6) $\varepsilon_{\text {voids }}=\frac{V_{\text {voids }}}{V_{3 D, t o t}}$

and for the particle employed in this study (Fig. 2), was measured to be $11.7 \%$.

The total initial porosity, $\varepsilon_{3 D, T o t, 0}$, is treated as an input parameter at present, although in the future this value might be obtained from adsorption and porosimetry experiments. Based on the heating rate and coal type, $\varepsilon_{3 D, T o t, 0}$ was taken to be $68 \%$ [28]. Using this value in conjunction with Eq. (5), the porosity of the microporous regions, $\varepsilon_{\text {micro-solid, } 0}$, was calculated to be $63.8 \%$.

A schematic of the distribution of porosity for each of the models is shown in Fig. 3 . The total porosity is the same in both models, but while the effective-continuum model treats the porosity as uniformly distributed, the pore-resolving model explicitly represents the porosity of the large pores/voids and consequently has a lower porosity associated with the surrounding char.

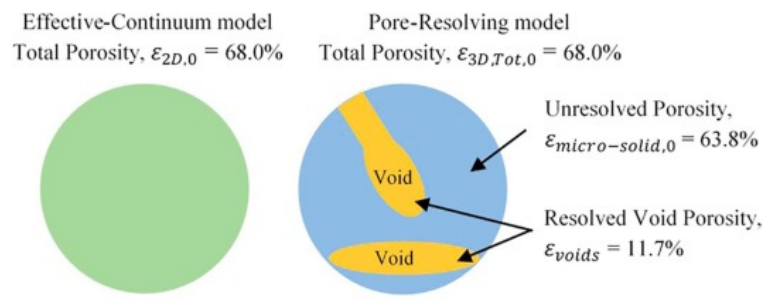

Fig. 3. Schematic of initial porosity distribution in effective-continuum (left) and pore-resolving (right) models.

To demonstrate that the mass of the effective-continuum and pore-resolving particles are identical, the masses are written in terms of the respective densities, char volumes and porosities:

(7a) $m_{2 D}=\rho_{t} V_{2 D}\left(1-\varepsilon_{2 D, 0}\right)$ 
(7b) $m_{3 D}=\rho_{t} V_{\text {micro-solid }}\left(1-\varepsilon_{\text {micro-solid }, 0}\right)$

where $\rho_{t}$, the true density, is defined as "density of the material excluding pores and voids" [26], and is identical in both models. Equating total porosities, $\varepsilon_{3 D, T o t, 0}$ and $\varepsilon_{2 D, 0}$, in Eq. (7a) and using Eq. (5) yields

(8) $m_{2 D}=\rho_{t} V_{\text {micro-solid }}\left(1-\varepsilon_{\text {micro-solid }, 0}\right)$

Since the particles were scaled to be have identical total volumes, $V_{2 D}$ may also be equated with $V_{3 D, t o t}$. Then using Eqs. (6), (2) leads to:

(9) $m_{2 D}=\rho_{t} V_{\text {micro-solid }}\left(1-\varepsilon_{\text {micro-solid, } 0}\right)$

which is identical to the expression for the mass of the pore-resolved particle in Eq. (7b), demonstrating that the masses of the two particles are identical.

Finally, because the initial specific surface area, $S_{g}$ (units of $\mathrm{m}^{2} / \mathrm{g}$ ), of the unresolved char regions is taken to be identical in both models, despite the slightly different porosities of the char regions, and the mass of char is identical in both models, the total initial surface area on which heterogeneous reactions can occur is also identical in both particles.

\subsection{Governing equations and Sub-models}

\subsubsection{Effective-continuum model}

Standard conservation equations for mass, momentum, species and thermal energy for laminar flow are solved in the boundary layer surrounding the particle, and are given by [29]:

(10) $\frac{\partial(\rho)}{\partial t}+\nabla \cdot(\rho \vec{v})=0$

(11) $\frac{\partial(\rho \vec{v})}{\partial t}+\nabla \cdot(\rho \vec{v} \vec{v})=-\nabla P+\nabla \cdot(\tilde{\tau})$

(12) $\frac{\partial\left(\rho Y_{i}\right)}{\partial t}+\nabla \cdot\left(\rho \vec{v} Y_{i}\right)=-\nabla \cdot \vec{J}$

(13) $\frac{\partial(\rho h)}{\partial t}+\nabla \cdot(\rho \vec{v} h)=\nabla \cdot\left[k \nabla T-\left(\begin{array}{ll}\sum_{i} & h_{i} J_{\iota}\end{array}\right)\right]$

where the variables are defined in the nomenclature. The governing equations account for advection and diffusion similar to the equations employed by Richter et al. [19] and Xue et al. [21], but also retain the unsteady terms. Radiation was not included in the simulations, as it was assumed that particle radiation is dominated by particle-to-particle (rather than particle-to-gas) radiation and that the surrounding particles are at the same temperature as the particle of interest [30]. Body forces were also ignored, as the particle was entrained in the surrounding flow. Homogeneous reactions were not included in the simulations, so species and energy source terms are not present for the fluid regions. 
Within the porous particle, classical upscaled, effective-continuum versions of the governing equations are employed to account for the presence of both fluid and solid phases [29], with the variables defined in the nomenclature:

$$
\begin{aligned}
& \text { (14) } \frac{\partial(\varepsilon \rho)}{\partial t}+\nabla \cdot(\varepsilon \rho \vec{v})=S_{m} \\
& \text { (15) } \frac{\partial(\varepsilon \rho \vec{v})}{\partial t}+\nabla \cdot(\varepsilon \rho \vec{v} \vec{v})=-\varepsilon \nabla P+\nabla \cdot(\varepsilon \bar{\tau})-\left(\frac{\varepsilon^{2} \mu}{B} \vec{v}\right) \\
& \text { (16) } \frac{\partial\left(\varepsilon \rho Y_{i}\right)}{\partial t}+\nabla \cdot\left(\varepsilon \rho \vec{v} Y_{i}\right)=-\nabla \cdot \vec{J}_{i}+R_{i} \\
& \text { (17) } \left.\frac{\partial\left(\varepsilon \rho h+(1-\varepsilon) \rho_{s} h_{s}\right)}{\partial t}+\nabla \cdot(\varepsilon \rho \vec{v} h)=\nabla \cdot\left[\begin{array}{ll}
k_{e f f} \nabla T-\left(\sum_{i}\right. & h_{i} \\
i
\end{array}\right)\right]+S_{f}^{h}
\end{aligned}
$$

A source term is included in the mass conservation equation (Eq. (14)) because heterogeneous reactions generate gaseous species within the porous solid, resulting in Stefan flow. The momentum equation (Eq. (15)) incorporates a viscous resistance term to account for the presence of the porous medium. Heterogeneous reactions occur within the unresolved porous regions and contribute volumetric species and energy source terms, $R_{i}$ and $S_{f}^{h}$. Local thermodynamic equilibrium between the solid and gas phases is assumed in the thermal energy equation (Eq. (17)). Sub-models required for the conservation equations are discussed in Sections $\underline{3.3}$

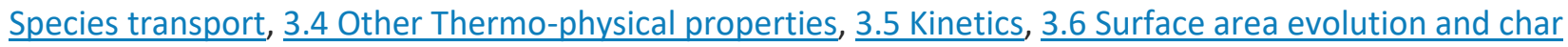
conversion.

As described in Section 1 , the use of upscaled, effective-continuum conservation equations throughout the particle presumes that the length-scale constraints of Eq. (1) are satisfied everywhere in the particle. This assumption will be evaluated by comparing the effective-continuum and pore-resolving approaches.

\subsubsection{Pore-resolving model}

For the pore-resolving model, conservation equations based on first principles (Eqs. (10), (11), (12), (13)) are solved not only in the surrounding gas boundary layer, but also in the resolved voids within the char particle, because the length-scale constraints (Eq. (1)) for upscaling are not satisfied for averaging over large heterogeneities. Within the microporous solid grains surrounding the resolved voids, effective-continuum conservation equations (Eqs. (14), (15), (16), (17)) are employed because the constraints for upscaling are satisfied in those regions.

To justify the use of a conservation equation approach in the resolved pores, the Knudsen number was calculated and found to vary between 0.0039 and 0.0066 . These values were determined by finding the maximum and minimum mean free paths for the four gas species present at 20 bar and $1800 \mathrm{~K}$ (see Section $\underline{3.7}$ ), which varied between $33 \mathrm{~nm}$ for $\mathrm{H}_{2}$ and $20 \mathrm{~nm}$ for $\mathrm{CO}$. The Knudsen number was then calculated using a conservative length scale of $5 \mu \mathrm{m}$ for the resolved pores in the $100 \mu \mathrm{m}$ particle. The fact that the Knudsen number was much smaller than unity justifies a conservation equation-based approach.

\subsection{Species transport}

The diffusive flux in the gas boundary layer and in the resolved voids is related to the mixture-averaged diffusion coefficient and the thermal diffusion coefficient of species $i$, calculated by kinetic theory, by

(18) $\vec{J}_{\iota}=-\rho D_{i, m} \nabla Y_{i}-D_{T, i} \frac{\nabla T}{T}$ 
Diffusion in porous media occurs through molecular diffusion as well as Knudsen diffusion. The Knudsen diffusivity is given by [31]:

(19) $D_{i, m, k n u d}=\frac{d_{p}}{3} \sqrt{\frac{8 R_{u} T}{\pi M W_{i}}}$

where the average pore diameter, $d_{p}$, is given in terms of the porosity, $\varepsilon$, and internal surface area, $S:($

$20 d_{p}=\frac{4 \varepsilon}{S}$

The expression for effective diffusivity in the effective-continuum regions incorporates both forms of diffusive $\underline{\text { transport }}$ and is given by [32]:

(21) $\frac{1}{D_{i, m, e f f}}=\frac{\varepsilon}{\tau}\left(\frac{1}{D_{i, m, k n u d}}+\frac{1}{D_{i, m}}\right)$

where the tortuosity, $\tau$, was assumed to be the inverse of porosity [33]. The diffusive flux expression in Eq. (18) is also employed for the porous char regions, with $D_{i, m}$ replaced by $D_{i, m, e f f}$. More complex transport models such as the Dusty Gas Model could be explored in the future. Finally, the permeability of the effectivecontinuum regions is given by [31]:

(22) $B=\frac{d_{p}^{2}}{32}$

\subsection{Other Thermo-physical properties}

The gas density was calculated from the ideal gas equation of state:

(23) $\rho=\frac{P}{R_{u} T \sum_{i} \frac{Y_{i}}{M W_{i}}}$

The viscosity and thermal conductivity for individual species were determined using kinetic theory, with values for the mixture given by [29]:

(24) $\mu=\sum_{i} \quad \frac{x_{i} \mu_{i}}{\sum_{j} x_{j} \emptyset_{i j}}$

(25) $k=\sum_{i} \frac{x_{i} k_{i}}{\sum_{j} x_{j} \emptyset_{i j}}$

(26) $\emptyset_{i j}=\frac{\left[1+\left(\frac{\mu_{i}}{\mu_{j}}\right)^{\frac{1}{2}}\left(\frac{M W_{, j}}{M W_{, i}}\right)^{\frac{1}{4}}\right]^{2}}{\left[8\left(1+\frac{M W_{, i}}{M W_{, j}}\right)\right]^{\frac{1}{2}}}$

The specific heat for each species, $i$, was calculated using a fourth-order polynomial in temperature, and the mixture specific heat was determined using a weighted mass fraction mixing law.

\subsection{Kinetics}

Char gasification kinetics are represented by two global heterogeneous reactions 
(R1) $\mathrm{C}+\mathrm{CO}_{2} \rightarrow 2 \mathrm{CO}$

(R2) $\mathrm{C}+\mathrm{H}_{2} \mathrm{O} \rightarrow \mathrm{CO}+\mathrm{H}_{2}$

Intrinsic (per unit internal surface area) power law gasification kinetics determined for a bituminous coal at high pressure were employed [34]:

(27) $\Re_{\mathrm{j}}=\mathrm{A}_{\mathrm{j}} \exp \left(-\frac{\mathrm{E}_{\mathrm{a}, \mathrm{j}}}{\mathrm{R}_{\mathrm{u}} \mathrm{T}}\right) P_{i}^{n}$

where $P_{i}$ is the partial pressure of species $i$. The rates were measured under Zone I conditions, free from transport effects. Although product inhibition is likely important under these conditions, we have opted for simple power-law expressions at present. The kinetic parameters are shown in Table 1.

Table 1. Gasification Reaction Rate Parameters [34].

\begin{tabular}{|l|l|l|}
\hline Reaction & $\mathrm{R} 1(\mathrm{j}=1)$ & $\mathrm{R} 2(\mathrm{j}=2)$ \\
\hline Pre-exponential factor, $A_{j}\left[\frac{\mathrm{g}}{\mathrm{m}^{2} s \mathrm{MPa}^{n}}\right]$ & $7.5 \times 10^{3}$ & $144.8 \times 10^{3}$ \\
\hline Activation energy $E_{a, j}\left[\frac{\mathrm{kJ}}{\mathrm{mol}}\right]$ & 200 & 212 \\
\hline Reaction order, $n$ & 0.41 & 0.41 \\
\hline
\end{tabular}

The species, mass and energy source terms in the effective-continuum conservation equations are related to the intrinsic reaction rates by

$$
\begin{aligned}
& \text { (28a) } R_{i}=\sum_{j} \quad \vartheta_{i, j} \frac{M W_{i}}{M W_{C}} S \mathcal{R}_{j} \\
& \text { (28b) } S_{m}=\sum_{i} \quad \sum_{j} \quad \vartheta_{i, j} \frac{M W_{i}}{M W_{C}} S \mathcal{R}_{j} \\
& \text { (28c) } S_{f}^{h}=-\sum_{j} \quad \Delta h_{r x n, j} \vartheta_{i, j} \frac{M W_{i}}{M W_{C}} S \mathcal{R}_{j}
\end{aligned}
$$

The internal surface area per unit volume, $S$ (see Eq. (29)) is related to the specific surface area, $S_{g o}$, also obtained from [34].

Finally, due to the high-temperature environment present during the devolatilization and char combustion stages of entrained flow gasification, thermal annealing would lead to a reduction in reactive sites and thus lower gasification reaction rates [35], modeled by a decrease in the pre-exponential factor, $A_{j}$ for both reactions [36], [37]. By comparing an assumed temperature profile for a particle moving through the gasifier to the experimental preparation conditions under which the kinetics were obtained, a pre-exponential factor reduction of $42.7 \%$ was calculated and applied to both reactions. As mentioned above, homogeneous reactions (e.g. water-gas shift) were omitted at present, to focus on the impact of the resolved morphological features on the interplay of heterogeneous reaction and transport.

\subsection{Surface area evolution and char conversion}

The evolution of the surface area $\left(\mathrm{m}^{2} \mathrm{c} / \mathrm{m}^{3}\right)$ of the effective-continuum regions with conversion follows the random pore model [38], [39] with the structural parameter, [Math Processing Error] $\psi$, set to zero for simplicity

(29) $S=S_{0}(1-X) \sqrt{1-\psi \ln (1-X)}$

Local char conversion, $X$, is related to the heterogeneous reaction rates, $\mathfrak{R}_{\mathrm{j}}$, by 
(30) $\frac{d X}{d t}=\frac{s}{\rho_{t}\left(1-\varepsilon_{0}\right)} \sum_{j=1}^{2} \quad \vartheta_{C, j} \mathfrak{R}_{\mathrm{j}}$

where $\vartheta_{C, j}$, is the stoichiometric coefficient of carbon (char) in heterogeneous reaction $j$. The local conversion, $X$, is linearly related to the local porosity, $\varepsilon$

(31) $X=\frac{\varepsilon-\varepsilon_{0}}{1-\varepsilon_{0}}$

and the conversion rate is related to the rate of change of porosity by

(32) $\frac{d X}{d t}=\frac{1}{1-\varepsilon_{o}} \frac{d \varepsilon}{d t}$

It is noted that $\varepsilon_{0}$ in Eq. (31) is defined as, $\varepsilon_{2 D, 0}$ and $\varepsilon_{\text {micro-solid,0 }}$, for the effective-continuum and poreresolving models, respectively. The char particles in both models are assumed to maintain their structural integrity. Fragmentation, which often occurs at high levels of particle conversion [40], is neglected in the present simulations, which are ended at a conversion of $90 \%$.

\subsection{Simulation domain, boundary conditions and initial conditions}

The particles in both simulations were contained at the center of a spherical domain containing $\mathrm{CO}, \mathrm{CO}_{2}, \mathrm{H}_{2}$ and $\mathrm{H}_{2} \mathrm{O}$, with a diameter 20 times that of the entrained particles. Because the particles were assumed to be entrained in the surrounding flow, there was no imposed velocity at the boundary and the velocity was free to adjust itself according to the Stefan flow. Other far-field boundary conditions are shown in Table 2 and are based on the gasification region of a single-stage entrained flow gasifier [41]. Pressure at the far-field boundary was 20 bar, the temperature was fixed at $1800 \mathrm{~K}$ and the species mole fractions are as shown in Table 2 . For the effective-continuum model, symmetry conditions were imposed at the center of the spherical particle.

Table 2. Far-field boundary conditions.

\begin{tabular}{|l|l|}
\hline Temperature, $T[\mathrm{~K}]$ & 1800 \\
\hline Pressure, $P[$ bar $]$ & 20 \\
\hline Mole Fraction, $x_{\mathrm{H}_{2} \mathrm{O}}[-]$ & 0.21 \\
\hline Mole Fraction, $x_{\mathrm{H}_{2}}[-]$ & 0.12 \\
\hline Mole Fraction, $x_{\mathrm{H}_{2}}[-]$ & 0.28 \\
\hline Mole Fraction, $x_{\mathrm{CO}}[-]$ & 0.39 \\
\hline
\end{tabular}

For initial conditions, reactants were removed from the particles in order to prevent unrealistically high initial reaction rates caused by uniform reactant profiles throughout the particles. Species and temperature profiles adjust to their nearly quasi-steady profiles after only a few time steps, and the influence of the initial species profiles is minimal, typical of such systems [15].

\subsection{Discretization and mesh validation}

The mesh for the three-dimensional, pore-resolving simulation was generated using Simpleware FE Module's "+FE Free" meshing algorithm, employing tetrahedral mesh elements. The two-dimensional, effectivecontinuum mesh was generated using FLUENT's meshing algorithm and was composed of quadrilateral elements. It is noted that the spherically-symmetric effective-continuum model is really one-dimensional, but was developed in two dimensions in FLUENT (as a half-circle in an axisymmetric configuration) for consistency with the pore-resolving model. Similar element sizing conditions were used for both models, with the finest elements existing within the particle and near the particle-fluid interfaces, and growing coarser approaching the far-field boundary. The meshes are illustrated for both models in Fig. 4. 


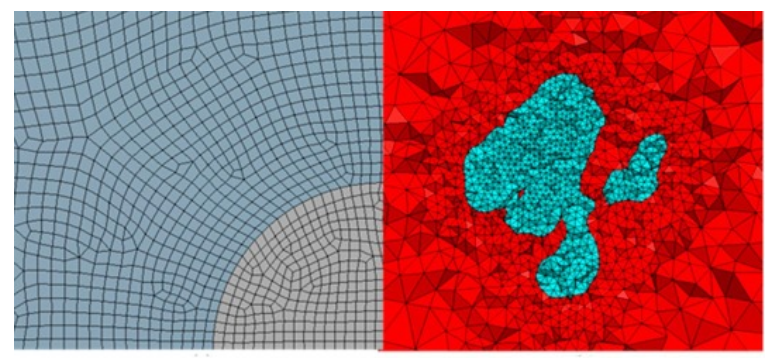

(a)

(b)

Fig. 4. Mesh, showing the particle and surrounding gas for (a) the spherical, effective-continuum model: light gray particle, dark gray gas, and (b) a plane from the 3-D, pore-resolving model: light blue particle, red voids/gas region.

A mesh study was conducted for both models to ensure that the solution was converged with respect to the mesh. Full transient simulations were run to 80 ms for meshes made up of varying numbers of elements and the results were qualitatively and quantitatively compared. The relative error for each mesh was calculated using char conversion volume averaged throughout the particle, taking the finest mesh as the "exact" value.

Conversion was chosen for quantitative comparison because it is influenced by both species and temperature profiles. The results of the convergence study are shown in Table 3. A mesh of 400,000 elements was deemed converged for the pore-resolving simulation and was employed for the results reported in Section 4 , while a mesh of 13,000 elements was used for the effective-continuum simulation.

Table 3. Relative error in char conversion as a function of mesh size.

\begin{tabular}{|l|l|l|l|}
\hline $\begin{array}{l}\text { Effective-Continuum Mesh Elements } \\
(2 \mathrm{D})\end{array}$ & Relative Error & $\begin{array}{l}\text { Pore-resolved Mesh Elements } \\
\text { (3D) }\end{array}$ & Relative Error \\
\hline $8.8 \times 10^{4}$ & N/A & $2.9 \times 10^{6}$ & N/A \\
\hline $5.8 \times 10^{4}$ & -0.00027 & $4.0 \times 10^{5}$ & 0.00083 \\
\hline $1.3 \times 10^{4}$ & -0.00046 & $1.2 \times 10^{5}$ & 0.00266 \\
\hline & & $7.9 \times 10^{4}$ & 0.0015 \\
\hline
\end{tabular}

\subsection{Numerical approach}

ANSYS Fluent version 17.2 was used for both the pore-resolving and effective-continuum models. The pressurebased solver was used for this low speed flow problem, with the coupled algorithm chosen to solve the momentum and pressure-based continuity equations together [29]. The spatial discretization scheme was selected based on the mass transfer peclet number, which was much smaller than one, as the flow is diffusiondominated and scalar values vary nearly linearly between cell faces [42]. The power law scheme was selected for a majority of the spatial discretization schemes and PRESTO! was chosen for the pressure-based solver. Fully implicit time-stepping was used, with a timestep of $0.5 \mathrm{~ms}$ selected based on a time step convergence study analgous to the mesh convergence study. The convergence of the solution at each time step was determined using scaled residuals, and the convergence criteria was determined by the residual values at which particle averaged scalars attained a constant value [43]. The pore-resolving model required $48 \mathrm{~h}$ of run-time on a 28core Intel ${ }^{\circledR}$ Xeon ${ }^{\circledR}$ E5-2690 v4 processor operating @ $2.60 \mathrm{GHz}$, with 256 GB of RAM and a 64-bit operating system.

\section{Results and discussion}

Validation of the models with experimental data would require knowledge of physical parameters, such as the full pore size distribution, and kinetic input parameters, such as zone I reaction rate data, in addition to zone II measurements of conversion for this specific particle for validation. This data is not available at present. While the previously-mentioned idealized pore-resolving models attempted validation using a simulation of non- 
porous particles against experimental temperature data from a variety of coals [19], [21], such an approach was not deemed meaningful for this situation and validation with experimental data was not attempted. Because the goal of this paper is to propose a new modeling framework and to compare a morphologically-resolved model to an effective-continuum model of the same particle, a consistent comparison between the two approaches is sufficient for the present purposes.

To contrast the spherically-symmetric, effective-continuum model with the three-dimensional, pore-resolving model, the respective velocity vectors and magnitudes are shown in Fig. 5 for planes passing through the particle center, with the respective particle-boundary layer interfaces delineated in white. As there is no imposed velocity at the boundary, the outward velocity is due to Stefan flow caused by the heterogeneous reactions. For the spherically-symmetric model (Fig. $5 \mathrm{a}$ ), the maximum velocity occurs just outside the interface and decreases toward the particle center. For the pore-resolving, three-dimensional model (Fig. $5 \mathrm{~b})$, the velocity field was highly asymmetric due to the irregular particle morphology and the magnitude of the velocity maxima were larger than those predicted by the effective-continuum model. The velocity maxima generally occur at the outer parts of the particle-boundary layer interface in the pore-resolving model, but local maxima are also observed within the resolved voids, where channeling occurs. The upper inset in Fig. $5 \mathrm{~b}$ illustrates the threedimensional character of the flow field.

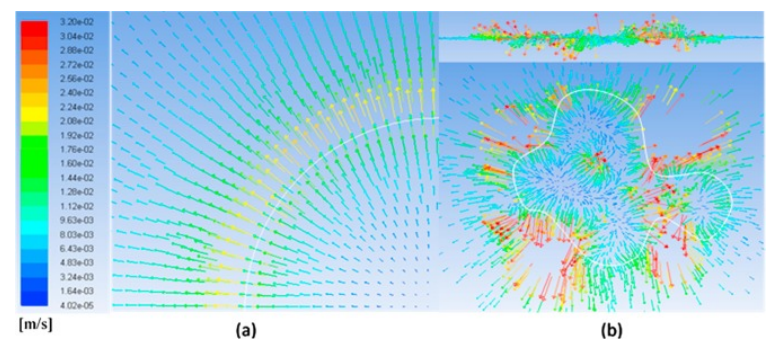

Fig. 5. Velocity vector field at 25ms: (a) effective-continuum model, and (b) pore-resolving model, with a second view in a plane orthogonal to the first (upper inset).

Fig. 6 shows mole fraction contours of reactants $\mathrm{H}_{2} \mathrm{O}$ and $\mathrm{CO}_{2}$ within the particles at $25 \mathrm{~ms}$ for planes passing through the particle centers, for the effective-continuum and pore-resolving simulations, with the respective particle-boundary layer interfaces delineated in white. It is noted that voids exist within the white outline for the pore-resolving model. Reactant penetration is asymmetric and significantly higher in the pore-resolving model, for both $\mathrm{H}_{2} \mathrm{O}$ and $\mathrm{CO}_{2}$, and mole fractions are closer to the values at the far-field boundary (the scales bars are identical for both simulation). 


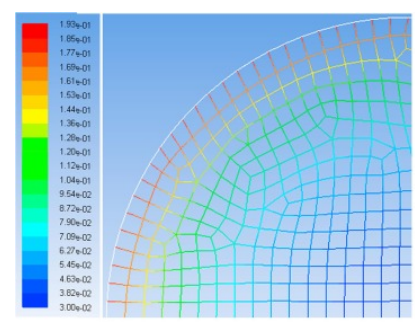

(a)

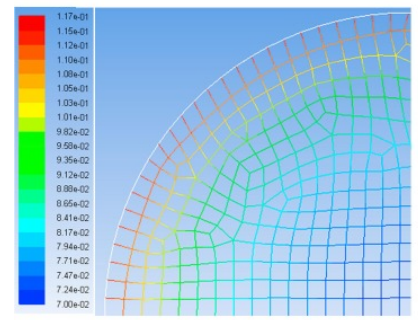

(c)

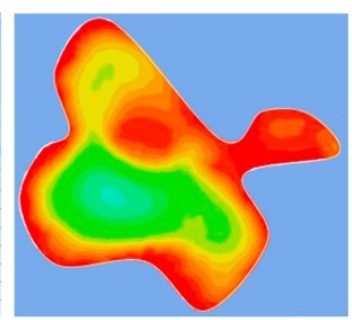

(b)

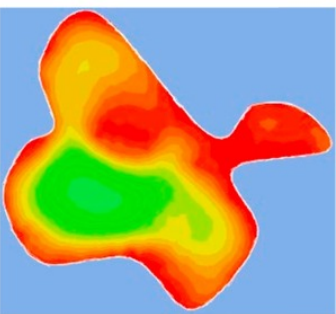

(d)

Fig. 6. Reactant mole fraction contours within the particles at $25 \mathrm{~ms}$ : (a) $\mathrm{H}_{2} \mathrm{O}$, effective-continuum model, (b) $\mathrm{H}_{2} \mathrm{O}$, pore-resolving model, (c) $\mathrm{CO}_{2}$, effective-continuum model, (b) $\mathrm{CO}_{2}$, pore-resolving model.

The qualitative differences observed in Fig. 6 are quantified in Fig. 7, in which profiles of reactant mole fraction are plotted as a function of particle radius. For the spherically-symmetric, effective-continuum model, the data was simply taken along the particle's axis (as all lines from the particle center to edge yield identical results). To generate corresponding radial profiles for the three-dimensional, pore-resolving model, data was averaged over azimuthal and polar directions at every radial position using:

(33) $x_{i, 3 D}(r)=\frac{1}{4 \pi r^{2}} \int_{0}^{2 \pi} \int_{0}^{\pi} x_{i}(r, \theta, \emptyset) r^{2} \sin \theta d \emptyset d \theta$
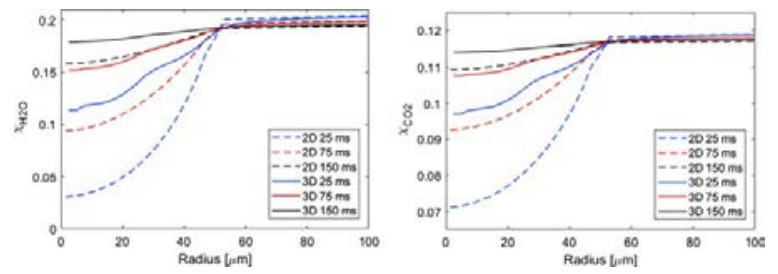

Fig. 7. Radial profiles of reactant mole fraction at three times for the effective-continuum (2D) and poreresolving (3D) models, for (a) $\mathrm{H}_{2} \mathrm{O}$, and (b) $\mathrm{CO}_{2}$.

The present averaging is performed over both the resolved voids and the microporous grains, to provide a meaningful comparison with the effective-continuum model, which lumps both regions into an effective porous continuum.

Fig. 7 shows reactant mole fractions as a function of radius for both the effective-continuum ("2D”) and poreresolving models ("3D") at three times. The particle domain extends to a radius of $50 \mu \mathrm{m}$, and a portion of the gas boundary layer (up to $100 \mu \mathrm{m}$ ) is also shown in the figures. The mole fraction of $\mathrm{H}_{2} \mathrm{O}$ vapor is lower than that of carbon dioxide within both particles because reaction (R2) is faster than reaction (R1). It is noted from Fig. 7 that the mole fraction profiles in the effective-continuum model decrease smoothly and monotonically with radius, as would be expected from solving reaction-diffusion equations on a spherically symmetric domain. The pore-resolving model, however, had more irregular profiles and almost deviated from monotonicity, due to the non-homogeneous distribution of the resolved voids within the particle and the elevated reactant concentration within those voids. 
Fig. 7 shows that the pore-resolving model's reactant mole fractions were always higher throughout the particle, for both $\mathrm{H}_{2} \mathrm{O}$ and $\mathrm{CO}_{2}$, than the effective-continuum model. This is partially because the resolved voids serve as channels that enhance reactant transport throughout the particle and increase its concentration compared to the effective-continuum model. Furthermore, the enhanced reactant concentration within the voids acts to decrease the characteristic length-scale for diffusion within the microporous solid grains. Whereas the characteristic length-scale for diffusion in the effective-continuum model is the radius of the entire particle, the characteristic length-scale for diffusion in the pore-resolving model is the local half-width of a microporous solid grain, which separates voids that contain reactant at concentrations not dissimilar from the far-field boundary.

The contribution of this second, less-obvious mechanism can be demonstrated by examining Fig. 8 , which compares radial reactant profiles for the effective-continuum model ("2D"), for the pore-resolving model averaged over all regions as in Eq. (33) and Fig. 7 ("3D"), and additionally, for the pore-resolving model averaged over azimuthal and polar directions only within the microporous solid regions ("3DMPS"), using

(34) $x_{i, 3 D, M P S}(r)=\frac{\int_{0}^{2 \pi} \int_{0}^{\pi} x_{i}(r, \theta, \emptyset) f(\varepsilon) r^{2} \sin (\theta) d \theta d \emptyset}{\int_{0}^{2 \pi} \int_{0}^{\pi} f(\varepsilon) r^{2} \sin (\theta) d \theta d \emptyset}$

where the function $f(\varepsilon)$ equals one for microporous solid regions and zero for void regions. Even within the microporous solid regions of the pore-resolving model, the reactant concentrations are significantly higher than in the effective-continuum model. This is despite the fact that the microporous grains in the pore-resolving model have a lower porosity, and thus a lower effective diffusivity, than the porous char in the effectivecontinuum model. This clearly establishes the importance of the reduced characteristic length-scale for diffusion within the microporous solid grains, which is captured by the pore-resolving model, but not by typical effectivecontinuum models. Effective-continuum models could potentially be adapted to account for this phenomenon in a general manner, as in [30], or using a similar "bidisperse macro- and micro-pore" approach [44].
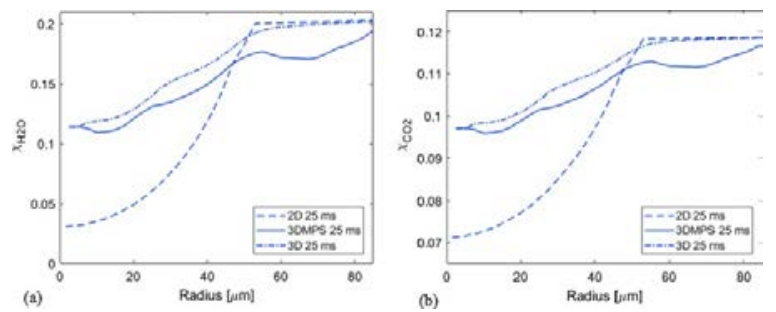

Fig. 8. Radial profiles of reactant mole fraction for the effective-continuum model (2D), the pore-resolving model averaged only over the microporous solid regions (3DMPS), and the pore-resolving model averaged over the resolved voids and the microporous solid regions (3D), for (a) $\mathrm{H}_{2} \mathrm{O}$, and (b) $\mathrm{CO}_{2}$.

Temperature contours for planes passing through the particle centers for the effective-continuum model and the pore-resolving model are shown in Fig. 9a and 9b, respectively, while Fig. $9 \mathrm{c}$ shows a three-dimensional surface distribution of temperature for the pore-resolving model. It is observed that there are regions of slightly higher temperature predicted by the pore-resolving model that are not captured by the effective-continuum model (the same scale is used throughout Fig. 9). 

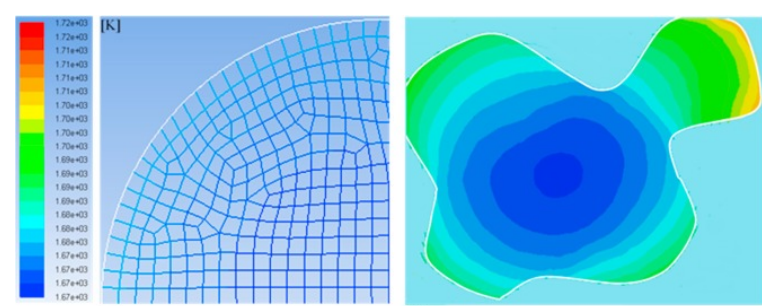

(a)

(b)

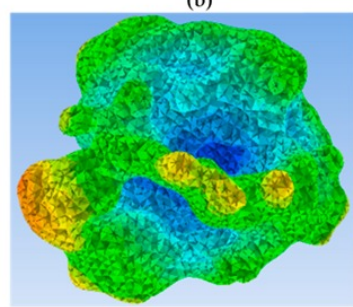

(c)

Fig. 9. Particle temperature contours at $25 \mathrm{~ms}$, for planes passing through the particle center for (a) the effective-continuum model, (b) the pore-resolving model, and (c) the three-dimensional surface temperature distribution for the pore-resolving model.

This qualitative observation is quantified in Fig. 10, which shows radial temperature profiles for the effectivecontinuum and pore-resolving models, with the latter again computed by averaging over polar and azimuthal directions at all radii (over both void and microporous char regions),

$$
\text { (35) } T(r)=\frac{1}{4 \pi r^{2}} \int_{0}^{2 \pi} \int_{0}^{\pi} T(r, \theta, \varnothing) r^{2} \sin \theta d \varnothing d \theta
$$

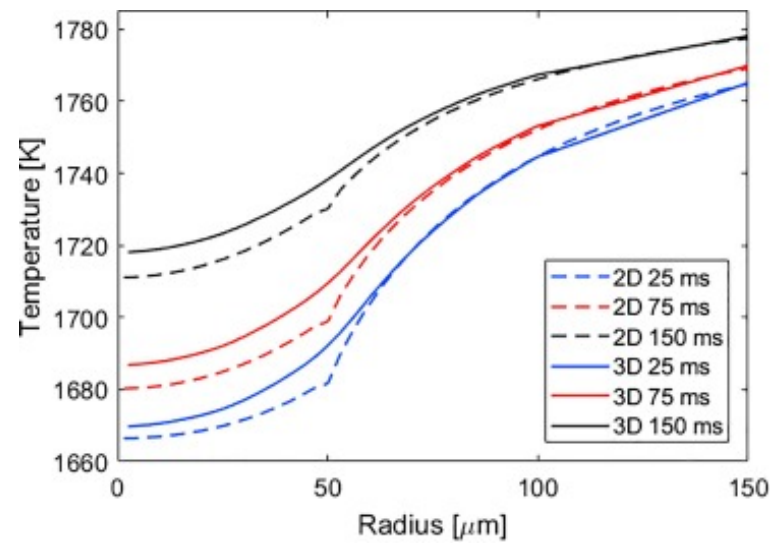

Fig. 10. Radial profiles of temperature at three times for effective-continuum (2D) and pore-resolving (3D) models.

Because gasification reactions are endothermic, higher reaction rates lead to lower temperatures in the char particle than in the external boundary layer. The temperature decrease was most severe for both models at early times, when reaction rates were fastest. Compared to the mole fraction profiles, the difference in temperature profiles between the two models is less significant, being less than $10 \mathrm{~K}$ at all positions and times. Nonetheless, the pore-resolving model has slightly higher temperatures throughout the particle than the effective-continuum model at all times, even though it experiences higher reaction rates than the effectivecontinuum model (see Fig. 11 below). This is attributed to the enhanced transport of hot reactant gas from the boundary layer into the voids, and the irregular, protruding shape of the particle, both of which also act to decrease the characteristic length-scale for heat transfer within the microporous grains. 


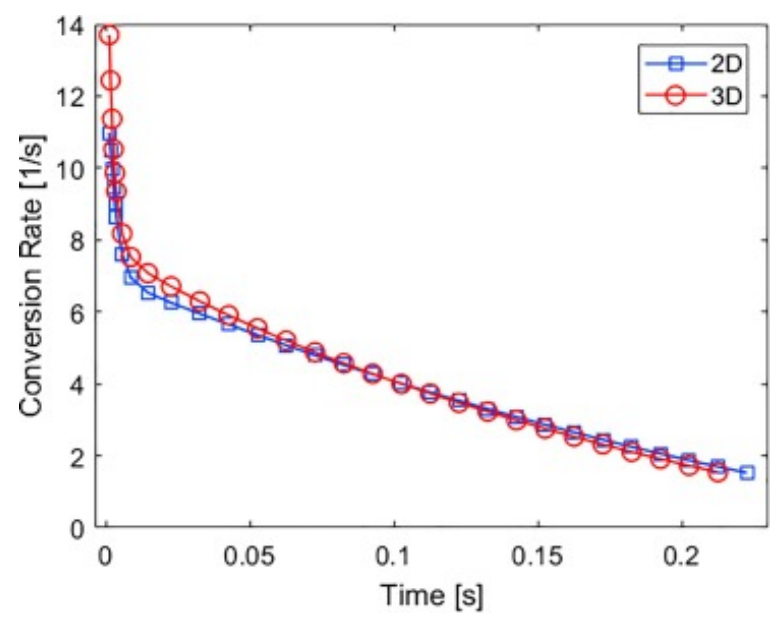

Fig. 11. Rate of char conversion vs. time for effective-continuum (2D) and pore-resolving (3D) models.

The total rate of char conversion, $d X / d t$, integrated over the entire particle, is compared in Fig. 11 for the poreresolving and effective-continuum approaches. The rate of char conversion is an important model output, as it is proportional to the species and energy source terms from sub-grid-scale particles into the gas-phase solver of reactor-scale CFD simulations. For both models, the internal surface area and reaction rates are highest early in conversion and decrease with time, since the pore structure parameter, $\psi$, is set to zero in Eq. (29). Fig. 11 shows that the pore-resolving model has a conversion rate that is up to $19.9 \%$ larger than the effectivecontinuum model, and that the rate of conversion remains higher in the pore-resolving model until $97.5 \mathrm{~ms}$, after which the effective-continuum model has a slightly higher conversion rate.

The enhanced reactant penetration captured by the pore-resolving model is the primary driver behind the differences in conversion rate for the two simulations. As discussed above, the large voids act as channels that increase reactant penetration throughout the pore-space of the particle. This, in effect, reduces the characteristic length-scale for diffusion from the characteristic size of the entire particle (as it is in effectivecontinuum approaches), to the characteristic size of the individual microporous grains (in pore-resolving models), since the voids surrounding each microporous region contain a gas concentration not dissimilar to that present outside the particle.

To explain the initial difference in char conversion rates predicted by the models and their subsequent similarity as time progresses (ii. 11), ex-post-facto effectiveness factors, $\eta$, were calculated for each model and for each heterogeneous reaction, $j$, by integrating the reaction rates over the entire volume of the char (the entire effective-continuum domain and the microporous solid regions of the pore-resolving model)

(36) $\eta_{j}=\frac{\iiint R_{j, \text { actual }} d V}{\iiint R_{j, \text { ideal }} d V}$

The actual reaction rates in Eq. (36) were calculated using the local, instantaneous values of reactant mole fractions in Eqs. (27), (28a), which are affected by transport limitations, while the ideal reaction rates in Eq. (36) employ the far-field mole fractions that would prevail throughout the entire particle in the absence of any transport limitations.

Fig. 12 shows that the $\mathrm{C}+\mathrm{CO}_{2}$ reaction (R1) occurs close to zone I conditions throughout the gasification process, while the $\mathrm{C}+\mathrm{H}_{2} \mathrm{O}$ reaction ( $\mathrm{R} 2$ ) is under zone II intra-particle diffusion control early in the conversion process. At later times, reaction (R2) also approaches zone I conditions, as the porosity and effective diffusivities increase, and the internal surface area decreases, both of which act to shift the process to kinetic control. For both reactions, and at all times, the pore-resolving simulation predicts higher effectiveness factors than the 
effective-continuum model, because it captures the enhanced reactant transport via the intra-particle voids, which reduces intra-particle diffusion limitations.

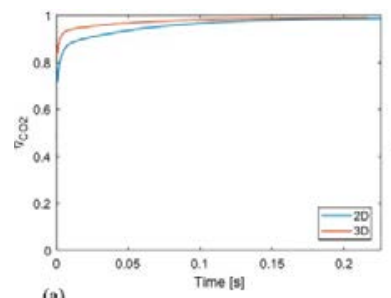

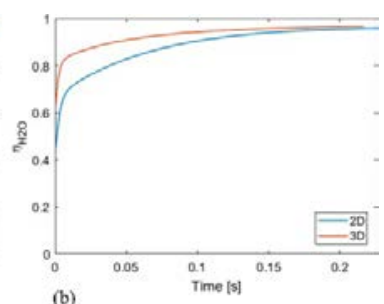

Fig. 12. Ex-post-facto effectiveness factor calculated by the effective-continuum model ("2D") and the poreresolving simulation ("3D"), for (a) the $\mathrm{C}+\mathrm{CO}_{2}$ reaction (R1) and (b) the $\mathrm{C}+\mathrm{H}_{2} \mathrm{O}$ reaction (R2).

The evolution of the $\mathrm{C}+\mathrm{H}_{2} \mathrm{O}$ effectiveness factor in Fig. 12 also explains the differences in predicted char conversion rates between the pore-resolving and effective-continuum models in Fig. 11. The $\mathrm{C}+\mathrm{H}_{2} \mathrm{O}$ reaction dominates char conversion because it is the faster reaction and because more $\mathrm{H}_{2} \mathrm{O}$ exists at the far-field boundary than $\mathrm{CO}_{2}$ in the present simulations. At early times, when this reaction occurs under zone II (intraparticle transport-limited conditions), the enhanced transport via the large macropores and voids affects the overall reaction rate and the pore-resolving model thus predicts the higher char conversion rates seen in Fig. 11. At later times, when both effectiveness factors approach unity and even the $\mathrm{C}+\mathrm{H}_{2} \mathrm{O}$ occurs near the zone $\mathrm{I}$, kinetically-limited regime, the impact of the enhanced transport via the large macropores and voids is negligible. Under such conditions, in which intra-particle transport is not limiting, the effective-continuum model and poreresolving model predict similar reaction rates.

\section{Conclusion}

A pore-resolving simulation approach based on micro-CT is proposed for zone II gasification and combustion of porous char particles. The simulation resolves the particle's large macropores, voids and fractures and solves conservation equations based on first principles in those regions, and employs upscaled, effectivecontinuum equations only in the micro- and meso-porous char surrounding the large voids. In contrast, the vast majority of existing, spatially-resolved, single particle models employ an effective-continuum treatment throughout the entire particle, treating all morphological features as sub-grid-scale, even though upscaling over regions containing large voids is not valid for zone II conditions.

The proposed pore-resolving approach was tested for entrained flow gasification conditions and the results were compared to a corresponding effective-continuum, spherically symmetric model for a char particle with the same initial mass, volume, equivalent diameter, total porosity and surface area. The presence of large macropores and voids was found to significantly enhance species transport through the char particle, not only between the particle surface and its nominal center, but also within the microporous solid grains surrounding the voids, compared to the effective-continuum particle. This finding indicates that for the weak zone II conditions tested, the true characteristic length-scale for diffusion is nearer to the length-scale of a microporous solid grain rather than the radius of the entire particle. This also implies that for the conditions tested, that the impact of the reduced porosity and diffusivity within the porous regions of the pore-resolved model, compared to the effective-continuum approach, is not as significant as the impact of the reduced characteristic lengthscale for diffusion.

The predicted rates of char conversion, which are the most important model outputs from the standpoint of reactor-scale CFD simulations, differ significantly early in conversion, but become similar soon thereafter, for the conditions tested. The early difference in reaction rates is attributed to the increased penetration of reactants captured by the pore-resolving model compared to the effective-continuum model prior to $25 \mathrm{~ms}$. 
As conversion proceeds, the internal surface area and reaction rates decrease, and the porosity and diffusivities increase, allowing the gasification reactions to transition from a weak zone II regime to nearly kineticallycontrolled zone I conditions, as calculated from ex-post-facto effectiveness factors. Under kinetic control, it is expected that the rates of conversion predicted by the two simulations would be nearly identical, given the fact that transport through the particle is no longer limiting in either model and the fact that the total internal surface areas are initially identical.

Pore-resolving simulation based on micro-CT is a novel, predictive and feasible approach to fundamentally understanding the interplay between reaction, transport and morphology during char gasification and combustion. Because differences between pore-resolving and effective-continuum approaches become more pronounced as intra-particle gradients become more important, the modeling framework shows promise for combustion and oxy-combustion conditions, which occur under stronger zone II behavior than gasification. Future work will also simulate a larger number of pulverized sized particles with varying morphologies, imaged with a higher resolution micro-CT, to draw statistically meaningful conclusions. The results, combined with characterization of char morphological distributions for particular coal type/reactor conditions, can be used to inform lower order models, such as effectiveness-factor models, which are appropriate for reactor-scale CFD simulations.

\section{Acknowledgement}

Acknowledgment is made to the donors of The American Chemical Society Petroleum Research Fund for support of this research. Images used courtesy of ANSYS, Inc. The authors thank Dr. Taly Gilat Schmidt for performing the micro-CT imaging.

\section{References}

[1]S. Schulze, A. Richter, M. Vascellari, A. Gupta, B. Meyer, P.A. Nikrityuk Novel intrinsic-based submodel for char particle gasification in entrained-flow gasifiers: model development, validation and illustration Appl Energy, 164 (2016), pp. 805-814, 10.1016/j.apenergy.2015.12.018

[2] B.M. Franchetti, F. Cavallo Marincola, S. Navarro-Martinez, A.M. Kempf Large Eddy simulation of a 100 kWth swirling oxy-coal furnace Fuel, 181 (2016), pp. 491-502, 10.1016/i.fuel.2016.05.015

[3] G. Liu, S. Niksa Coal conversion submodels for design applications at elevated pressures. Part II. Char gasification Prog Energy Combust Sci, 30 (2004), pp. 679-717, 10.1016/i.pecs.2004.08.001

[4] E.S. Hecht, C.R. Shaddix, A. Molina, B.S. Haynes Effect of CO2 gasification reaction on oxy-combustion of pulverized coal char Proc Combust Inst, 33 (2011), pp. 1699-1706, 10.1016/j.proci.2010.07.087

[5] E.M. Hodge, D.G. Roberts, D.J. Harris, J.F. Stubington The significance of char morphology to the analysis of high-temperature char-CO2 reaction rates Energy Fuels, 24 (2010), pp. 100-107, 10.1021/ef900503x

[6] J. Auriault Upscaling heterogeneous media by asymptotic expansions J Eng Mech, 128 (2002), pp. 817-822

[7] S. Whitaker Diffusion and dispersion in porous media AIChE J, 13 (1967), pp. 420-427

[8] M. Sahimi, G.R. Gavalas, T.T. Tsotsis Statistical and continuum models of fluid-solid reactions in porous media Chem Eng Sci, 45 (1990), pp. 1443-1502

[9] Y. Bachmat, J. Bear Macroscopic modelling of transport phenomena in porous media. 1: the continuum approach Transp Porous Media, 1 (1986), pp. 213-240

[10] R. Sahu, R.C. Flagan, G.R. Gavalas Discrete simulation of cenospheric coal-char combustion Combust Flame, 77 (1989), pp. 337-346

[11] J. Yu, J.A. Lucas, T.F. Wall Formation of the structure of chars during devolatilization of pulverized coal and its thermoproperties : a review Prog Energy Combust Sci, 33 (2007), pp. 135-

170, 10.1016/j.pecs.2006.07.003 
[12] F. Boso, I. Battiato Homogenizability conditions for multicomponent reactive transport processes Adv Water Resour, 62 (2013), pp. 254-265

[13] E.M. Ryan, A.M. Tartakovsky, C. Amon Pore-scale modeling of competitive adsorption in porous media J Contam Hydrol, 120-121 (2011), pp. 56-78, 10.1016/j.jconhyd.2010.06.008

[14] M. Sahraoui, M. Kaviany Direct simulation vs volume-averaged treatment of adiabatic, premixed flame in a porous medium Int J Heat Mass Transf, 37 (1994), pp. 2817-2834

[15] S.L. Singer, A.F. Ghoniem Comprehensive gasification modeling of char particles with multi-modal pore structures Combust Flame, 160 (2013), pp. 120-137, 10.1016/j.combustflame.2012.09.007

[16] M. Loewenberg, Y.A. Levendis Combustion behavior and kinetics of synthetic and coal-derived chars : comparison of theory and experiment Combust Flame, 84 (1991), pp. 47-65

[17] R. Jovanović, E. Marek, S. Maletić, D. Cvetinović, Z. Marković Lattice Monte Carlo simulation of single coal char particle combustion under oxy-fuel conditions Fuel, 151 (2015), pp. 172-

181, 10.1016/j.fuel.2015.02.104

[18] H. Xin, C. Wang, E. Louw, D. Wang, J.P. Mathews Atomistic simulation of coal char isothermal oxy-fuel combustion: char reactivity and behavior Fuel, 182 (2016), pp. 935-943, 10.1016/i.fuel.2016.05.103

[19] A. Richter, P.A. Nikrityuk, B. Meyer Three-dimensional calculation of a chemically reacting porous particle moving in a hot 02/CO2 atmosphere Int J Heat Mass Transf, 83 (2015), pp. 244-

258, 10.1016/i.ijheatmasstransfer.2014.11.090

[20] K. Wittig, P. Nikrityuk, A. Richter Drag coefficient and Nusselt number for porous particles under laminar flow conditions Int J Heat Mass Transf, 112 (2017), pp. 1005-

1016, 10.1016/i.ijheatmasstransfer.2017.05.035

[21] Z. Xue, Q. Guo, Y. Gong, J. Xu, G. Yu Numerical study of a reacting single coal char particle with different pore structures moving in a hot 02/CO2 atmosphere Fuel, 206 (2017), pp. 381389, 10.1016/j.fuel.2017.06.035

[22] X. Ni, J. Miao, R. Lv, X. Lin Quantitative 3D spatial characterization and flow simulation of coal macropores based on $\boldsymbol{\mu C T}$ technology Fuel, 200 (2017), pp. 199-207, 10.1016/j.fuel.2017.03.068

[23] P.N. Ciesielski, M.F. Crowley, M.R. Nimlos, A.W. Sanders, G.M. Wiggins, D. Robichaud, et al. Biomass particle models with realistic morphology and resolved microstructure for simulations of intraparticle transport phenomena Energy Fuels, 29 (2015), pp. 242-254, 10.1021/ef502204v

[24] M.B. Pecha, M. Garcia-Perez, T.D. Foust, P.N. Ciesielski Estimation of heat transfer coefficients for biomass particles by direct numerical simulation using microstructured particle models in the laminar regime ACS Sustain Chem Eng, 5 (2017), pp. 1046-1053, 10.1021/acssuschemeng.6b02341

[25] Simpleware. ScanIP Software, Mountain View, CA, USA; 2017.

[26] J. Rouquerolt, D. Avnir, C.W. Fairbridge, D.H. Everett, J.H. Haynes, N. Pernicone, et al. Recommendations for the characterization of porous solids Pure Appl Chem, 66 (1994), pp. 1739-1758 doi:10.1351/pac199466081739

[27] L.S. Fan, Z. Chao [Size and properties of particles. princ. Gas-solid flows] Cambridge University Press (1998) p. 3-45

[28] K. Zygourakis Effect of pyrolysis conditions on the macropore structure of coal-derived chars Energy Fuels (1993), pp. 33-41

[29] ANSYS FLUENT Theory Guide V17.2 2016.

[30] Y. Cai, K. Zygourakis A multiscale transient model for combustion of highly porous chars Ind Eng Chem Res, 42 (2003), pp. 2746-2755

[31] R. Jackson Transport in porous catalysts Elsevier, New York (1977)

[32] C.N. Satterfield Mass transfer in heterogeneous catalysis (third ed.), M.I.T Press (1970)

[33] S.V. Sotirchos, N.R. Amundson Dynamic behavior of a porous char particle burning in an oxygencontaining environment. Part I. Constant particle radius AIChE J, 30 (1984), pp. 537-549 
[34] A. Tremel, H. Spliethoff Gasification kinetics during entrained flow gasification - Part II: Intrinsic char reaction rate and surface area development Fuel, 107 (2013), pp. 653-661, 10.1016/i.fuel.2012.10.053

[35] A. Zolin, A. Jensen, K. Dam-johansen Experimental study of char thermal deactivation Fuel, 81 (2002), pp. 1065-1075

[36] A. Zolin, A. Jensen Coupling thermal deactivation with oxidation for predicting the combustion of a solid fuel Combust Flame, 125 (2001), pp. 1341-1360

[37] E.M. Suuberg, M. Wojtowicz, J.M. Calo Some aspects of the thermal annealing process in a phenolformaldehyde resin char Carbon N Y, 27 (1989), pp. 431-440

[38] S.K. Bhatia, D.D. Perlmutter A random pore model for fluid-solid reactions : 1. Isothermal, kinetic control AIChE J, 26 (1980), pp. 379-386

[39] G.R. Gavalas A random capillary model with application to char gasification at chemically controlled rates AIChE J, 26 (1980), pp. 577-585

[40] B. Feng, S.K. Bhatia Percolative fragmentation of char particles during gasification Energy Fuels, 14 (2000), pp. 297-307

[41] C. Botero, R.P. Field, H.J. Herzog, A.F. Ghoniem Impact of finite-rate kinetics on carbon conversion in a high-pressure, single-stage entrained flow gasifier with coal-CO2 slurry feed Appl Energy, 104 (2013), pp. 408-417, 10.1016/j.apenergy.2012.11.028

[42] S. Patankar Numerical heat transfer and fluid flow Hemisphere Publishing, Washington D C (1980)

[43] ANSYS FLUENT User's Guide V17.2 2016.

[44] T. Dogu Diffusion and reaction in catalyst pellets with bidisperse pore size distribution Ind Eng Chem Res, 37 (1998), pp. 2158-2171 